\title{
Formation and Destruction of Thermo-Lipofuscin in Mitochondria
}

\section{Vekshin $\mathrm{NL}^{*}$ and Frolova MS}

Institute of Cell Biophysics, Russian Academy of Sciences, Pushchino, Moscow Region, 142290, Russia

*Corresponding author: Vekshin NL, Institute of Cell Biophysics, Russian Academy of Sciences, Pushchino, Moscow Region, 142290, Russia, Tel: 4967739432; Email: nvekshin@rambler.ru

Received Date: Jul 12, 2018; Accepted Date: Aug 9, 2018; Published Date: Aug 16, 2018

Copyright: @ 2018 Vekshin NL, et al. This is an open-access article distributed under the terms of the Creative Commons Attribution License, which permits unrestricted use, distribution, and reproduction in any medium, provided the original author and source are credited.

\begin{abstract}
Previously, we have demonstrated that moderate heating of mitochondria isolated from rat liver induces the formation of lipofuscin - a pigment of ageing. The resulting thermo-lipofuscin has a characteristic fluorescence intensity in the blue region $(460 \mathrm{~nm})$ upon excitation at the near UV $(360 \mathrm{~nm})$ like normal lipofuscin found in cells. In this paper, we studied the effect of various substances on the processes of formation and destruction of mitochondrial thermo-lipofuscin. Contrary to our expectations, anti-oxidants such as ionol, histidine and imidazole have accelerated the process of formation of thermo-lipofuscin in mitochondrial suspension. Thermo-lipofuscin has formed more slowly in a buffer of $\mathrm{pH}$ 6. Formation of thermo-lipofuscin has been reduced upon activation of mitochondrial respiration by succinate. The formed thermo-lipofuscin has been cleaved to some degree by reducing agents with an active sulfhydryl group - glutathione, cysteine, dithiothreitol. It can be assumed that one of the ways of formation of thermo-lipofuscin is oxidation of protein's SH-groups.
\end{abstract}

Keywords: Mitochondria; Lipofuscin; Anti-oxidants; Aging; Fluorescence; Super-oxide

\section{Introduction}

Mitochondria of normal mammalian cells age and are subsequently destroyed by lysosomal digestion in the process of mitophagy or by proteosomal lysis $[1,2]$. If the activity of intracellular lysosomes and proteasomes is not sufficient, mitochondria will degrade into lipofuscin (LF) granules. The main factors that determine the appearance of LF in cells are still not known in detail. One major factor is active oxygen species $[3,4]$.

LF is a high-polymer complex formed during degradation and aggregation of proteins, damaged by the products of lipid oxidation (4hydroxy-2-nonenal etc.) and sugars (e.g., glucose-6-phosphate). Its composition varies in different types of cells and organisms. Degraded proteins in LF are cross-linked as Schiff bases by both intra- and intermolecular covalent bonds. LF is resistant to degradation by proteolytic cell systems. This insoluble rubbish is not subjected to cell exocytosis [5]. It has a characteristic fluorescence band in the region of $460 \mathrm{~nm}$ upon excitation in the near UV [6].

There is a lot of data suggesting that the rate of LF formation under conditions of oxidative stress can be reduced by antioxidants. Among them the flavanols, polyphenols, catechins and oligomeric procyanidins are mentioned [7]. Curcumin inhibits the formation of malon-dialdehyde (a product of lipid peroxidation) and LF [8]. Tocopherols present in the medium also have an inhibitory effect on the accumulation of LF [9]. Lack of tocopherol enhances the rate of accumulation of LF, which is comparable with aging [10]. Addition of coenzyme Q10 (ubiquinone) to rats' diets reduced the LF content in hippocampus [11]. Beta-carotene reduced oxidative stress, improved glutathione metabolism and decreased the LF formation [12]. Administration of lipoic acid to aged rats led to a reduction of LF in discrete brain regions [13]. The supplementation of creatine has shown a marked neuron-protective effect and significantly decreased accumulation of LF in mouse models of neurodegenerative diseases (Parkinson's disease, Huntington's disease, amyotrophic lateral sclerosis) [14]. Glutathione performs antioxidant function in a cell, and its deficiency leads to an increase in the amount of LF [15].

Antioxidants are able to stop or slow down the process of the LF formation. However, in many cases, LF granules of aging body occupy up to $70 \%$ of cell volume that leads to insufficient cellular actions. In such cases, it is necessary to remove the accumulated LF from the cytoplasm. Therefore, the aim of our work is not only to test the effect of a number of antioxidants on the formation of thermo-LF in isolated mitochondria, but also to search for the substances capable of destroying it.

\section{Materials and Methods}

\section{Chemicals and reagents}

$\beta$-nicotinamide adenine dinucleotide (NAD), L-glutathione reduced, L-cysteine, imidazole and dithiothreitol were supplied by ApplChem (Germany). Sodium succinate, isopropyl alcohol and Lhistidine were purchased from Panreac (Spane). Dimethyl sulfoxide (DMSO), sodium dodecyl sulfate (SDS) and other chemicals were obtained from Sigma-Aldrich (Germany). All reagents and chemicals were of the best commercial grade.

\section{Animal care and preparation}

Wistar rats (220-350 g) were obtained from the Nursery of laboratory animals of Institute Cell Biophysics (Pushchino). Animal care and experimental procedures were approved in accordance with the National Institutes of Health $(\mathrm{NIH})$ "Guide for the Care and Use of Laboratory Animals" (published by the National Academy Press, Washington, DC, 2011). 


\section{Isolation of rat liver mitochondria}

Mitochondria from rat liver were isolated in medium, containing 20 $\mathrm{mM}$ Tris- $\mathrm{HCl}$ and $250 \mathrm{mM} \mathrm{NaCl}(\mathrm{pH} 7.7)$ at $2^{\circ} \mathrm{C}$ by standard method with some modifications [16]. First, the initial suspension was centrifuged at BECKMAN J 2-21 at $1000 \mathrm{~g}$ during $15 \mathrm{~min}$. As a result, cells and other large particles were sedimented and discarded. The supernatant was centrifuged for $15 \mathrm{~min}$ at $5000 \mathrm{~g}$, leading to the precipitation of mature heavy mitochondria, which were then resuspended in the same medium $(9 \mathrm{ml}$ per $1.5 \mathrm{ml}$ of pellet) and dosed into 1-ml Eppendorf tubes, which were frozen and then were used for experiments (after defrosting).

It was decided to replace sucrose in the incubation buffer with 100 $\mathrm{mM} \mathrm{NaCl}$, because sucrose increased the base level of LF by $20 \%$. All mitochondrial fractions were aligned on protein content by incubation buffer $(100 \mathrm{mM} \mathrm{NaCl}$ and $10 \mathrm{mM}$ Tris-phosphate, $\mathrm{pH}$ 7.0) to a final concentration of $1 \mathrm{mg} / \mathrm{ml}$. Concentration of mitochondrial protein was determined by UV-express method [17].

\section{Termo-lipofuscin formation}

The formation of thermo-LF in mitochondrial suspension (1 $\mathrm{mg} / \mathrm{mL}$ ) was induced by 3 -hours heating in thermostat at $49^{\circ} \mathrm{C}$.

The obtained LF was detected by the appearance of a typical fluorescence band in the region at $460 \mathrm{~nm}$ with excitation at $360 \mathrm{~nm}$. The intensity of the fluorescence of endogenous pyridine nucleotides was negligibly small (since they were oxidized after freeze-thaw of mitochondria).

The destruction of the obtained LF by tested compounds was detected as the disappearance of the fluorescence band at $460 \mathrm{~nm}$.

All obtained samples were treated by SDS-a detergent that transforms a turbid mitochondrial suspension into a transparent solution.

Fluorescence spectra of all samples were recorded by upgraded spectrofluorimeters Perkin-Elmer MPF-44B (USA) and Carry ECLIPSE (USA) at $20^{\circ} \mathrm{C}$ in mirror cuvettes, which allowed amplify the signal by several times [18].

\section{Data analysis}

The means and standard deviations were obtained from at least three batches of mitochondria isolated from three-five different Wistar rats. The difference among treatment groups were analyzed by one-way or two-way ANOVA test. A p value less than 0.05 was considered statistically meaningful. Data processing was performed using the MS Office Excel 2015 and Origin Pro 8.5. All shown spectra were corrected on background (the buffer) and light-scattering.

\section{Results and Discussion}

One of the important properties of LF is its intense fluorescence in the blue region $[1,19,20]$ at the excitation in the near UV region. In our experiments, we observed the occurrence of intense fluorescence of LF after prolonged moderate heating $\left(3\right.$ hours at $49^{\circ} \mathrm{C}$ ) of a suspension of mitochondria from rat liver (Figure 1).

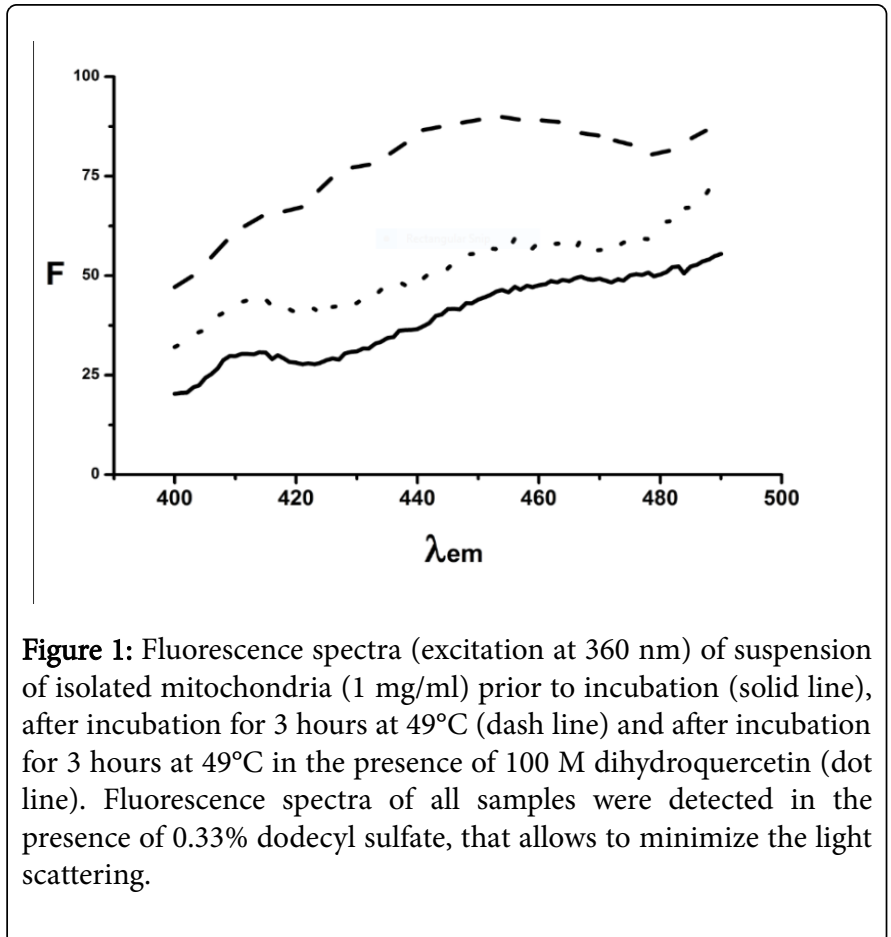

The temperature of $49^{\circ} \mathrm{C}$ is comfortable and conditional for acceleration the vibrations in proteins and lipids and for increase in velocity of all reactions. This temperature makes the proteins and lipids more accessible for oxidation by ROS. It is important that $49^{\circ} \mathrm{C}$ is only on 10 degree above than a normal temperature of a rat. Raising the temperature to this value in vitro accelerates the process of lipofuscin formation. On the other hand, this temperature does not lead to strong protein denaturation and aggregation.

A band with a maximum at $360 \mathrm{~nm}$ in the excitation spectrum was detected (not shown in figure). It should be noted that a small amount of LF is present in the mitochondrial suspension, even before heating. The tested substances were added to the mitochondrial suspension and incubated at $49^{\circ} \mathrm{C}$ for 3 hours. Under these conditions, partial denaturation of proteins, their aggregation and covalent cross-linking, including Schiff bases occurred in the forming LF.

The process of LF formation depends on the lipid peroxidation and therefore, in principal, is able to be terminated by antioxidants. In our experiments, several natural antioxidants-dihydroquercetin, glutathione, cysteine, imidazole, histidine, and synthetic antioxidant ionol were tested.

Dihydroquercetin that belongs to a class of bioflavonoids, was shown to have the highest anti-LF activity. It inhibits the LF formation by $80 \%$ during incubation at a concentration of $100 \mu \mathrm{M}$ with a suspension of mitochondria. A synthetic ionol (butylated hydroxytoluene-BHT), at a concentration of $100 \mu \mathrm{M}$ has not shown any positive effect. On the contrary, it accelerated the process of LF formation by $15 \%$. 
Citation: Vekshin NL, Frolova MS (2018) Formation and Destruction of Thermo-Lipofuscin in Mitochondria. Biochem Anal Biochem 7: 357. doi: $10.4172 / 2161-1009.1000357$

Page 3 of 5

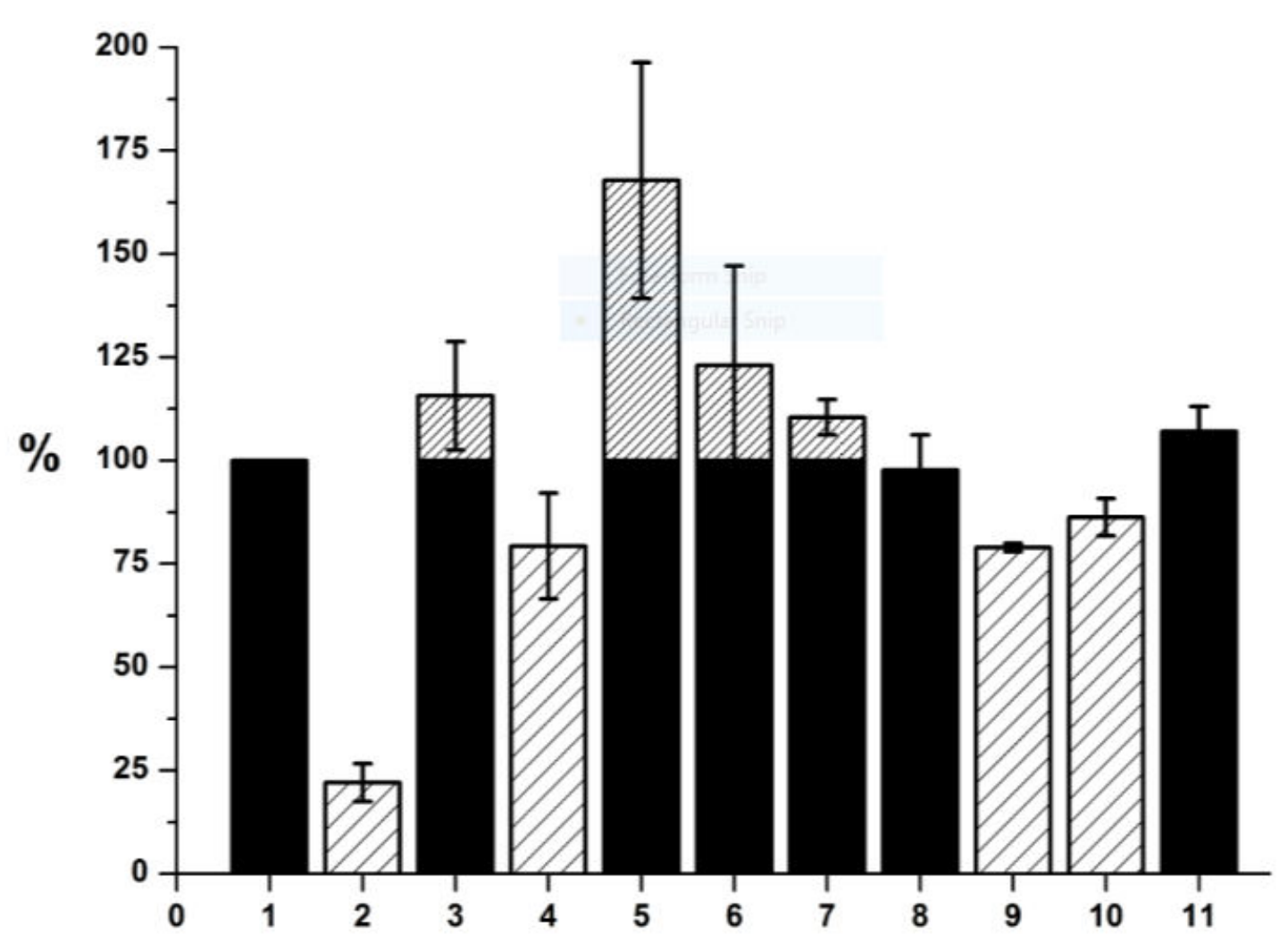

Figure 2: Formation of thermo-lipofuscin (in \%) in suspension of isolated mitochondria $\left(1 \mathrm{mg} / \mathrm{ml}\right.$ ) during incubation for 3 hours at $49^{\circ} \mathrm{C}$ without additions (1), with $100 \mu \mathrm{M}$ dihydroquercetin (2), $100 \mu \mathrm{M}$ ionol (3), $100 \mu \mathrm{M}$ reduced glutathione (4), $1 \mathrm{mM}$ cysteine (5), $1 \mathrm{mM}$ imidazole (6), $1 \mathrm{mM}$ histidine (7), $100 \mu \mathrm{M}$ NAD (8), $100 \mu \mathrm{M}$ succinate (9), without additions at $\mathrm{pH} 6$ (10), at $\mathrm{pH} 8$ (11).

It is known that imidazole and amino acid histidine that contains imidazole group, play an important role in the treatment of premature aging $[21,22]$. Histidine is capable of entering into redox reactions with superoxide and iron, which are pro-oxidants [23]. However, in our experiments, histidine and imidazole have not shown their antioxidant properties, but, on the contrary, caused an increase in the LF production. During incubation of mitochondria with $1 \mathrm{mM}$ imidazole and $1 \mathrm{mM}$ histidine for 3 hours at $49^{\circ} \mathrm{C}$ the quantity of LF increased by $23 \%$ and $10 \%$, respectively (Figure 2 ). In fact, imidazole ring is able to form Schiff bases [24]. It is possible that the increase in the quantity of LF in the suspension occurred due to this reason.

Reduced glutathione is one of the most important members of the antioxidant system cells. Its concentration in cells, as is known, is about $3 \mathrm{mM}$. In our experiments, its effect was dependent on the concentration. At a concentration of $1 \mathrm{mM}$ during incubation with mitochondria for 3 hours at $49^{\circ} \mathrm{C}$, the antioxidant effect was not noticeable, within experimental error. At a concentration of $100 \mathrm{mM}$, reduced glutathione decreased the amount of LF formed by $20 \%$.

Precursor of glutathione-cysteine (sulfur containing amino acid)-in principle, may also possess antioxidant activity. However, during incubation of mitochondrial suspension with $100 \mu \mathrm{M}$ or $1 \mathrm{mM}$ cysteine an increase in the quantity of LF was observed by $52 \%$ and $68 \%$ respectively. Cysteine appears to act as a pro-oxidant at these concentrations.
Such substances as cysteine, histidine and imidazole are not 100\%th antioxidants. They can act as pro-oxidants at high concentrations. Probably this is due to a reduction of iron atoms, which then come into the Fenton's reaction. This reaction starts the lipid per-oxidation and, as a consequence, the quantity of LF increases.

During the incubation of mitochondria with $1 \mathrm{mM}$ dithiothreitol, a decrease in the quantity of LF by $43 \%$ was observed. Presumably, dithiothreitol can react as an antioxidant or directly destroy S-S links and Schiff bases during the process of incubation of the suspension at $49^{\circ} \mathrm{C}$.

One of the main well-known generators of superoxide in the electron transport chain of mitochondria is $\mathrm{NADH}$, which is oxidized by the mitochondrial NADH dehydrogenase. Generation of superoxide can accelerate the loss of the enzyme's coenzyme flavin mononucleotide (FMN). In our previous work, we showed a possibility of decreasing the superoxide action by stabilizing $\mathrm{NADH}$ dehydrogenase by NAD [25]. Possibly, NAD is embedded in the second active site of the enzyme, thus preventing the exit of FMN from the active center into the free solution [26]. It might be assumed that the incubation of the mitochondrial suspension with NAD will decrease the LF production. However, the obtained result was almost the same as the control. Perhaps, the loss of FMN from the enzyme does not lead to an increase in the formation of LF. 
The thermal incubation of mitochondria with succinate accelerates their respiration and thereby decreases the oxygen content in the suspension (in a hermetic cuvette). This results in a significant reduction of lipid peroxidation. In our experiments, this correlates with a decrease in the LF formation by $21 \%$ compared to the control.

Apart from antioxidants and reducing agents, the formation of LF can be controlled by changing the $\mathrm{pH}$. It is known that superoxide radical anion at acidic $\mathrm{pH}$ values may be protonated with formation of a more reactive peroxyl radical, which is a weak acid. In our experiments, in order to create conditions at which the superoxide action is enhanced, a more acidic $\mathrm{pH}$ of 6.0 was used. However, it did not increase the number of LF after incubation of mitochondrial suspension. On the contrary, at $\mathrm{pH} 6.0$ the quantity of LF decreased by $14 \%$, compared to control (Figure 2). With increasing $\mathrm{pH}$ to 8.0 the quantity of LF was within experimental error, compared to the control (Figure 2).

After incubation of mitochondrial suspension at $49^{\circ} \mathrm{C}$ for 3 hours the quantity of LF is generally increased by an average of $53 \%$. Glutathione, cysteine and dithiothreitol were added to a suspension of mitochondria in order to test their ability to cleave the Schiff bases of thermal-LF. The LF degradation was detected by a reduction in the fluorescence at $460 \mathrm{~nm}$ (excitation-360 nm).

In addition to the antioxidant function, glutathione performs its primary function-the restoration of S-S links in proteins. Moreover, glutathione is able to cleave the Schiff bases. It was demonstrated in the reaction of glutathione with $\mathrm{N}, \mathrm{N}^{\prime}$-bis (2-pyridylmethylene)-1-4butanediamine (N,N',N",N"')-Cu(II)-diperchlorate (CuPUPY) [27]. In our experiments, this ability of glutathione was confirmed. After 1 hour incubation of mitochondrial thermo-LF (appeared in mitochondria after heating for 3 hours at $49^{\circ} \mathrm{C}$ ) with $1 \mathrm{mM}$ glutathione at $23^{\circ} \mathrm{C}$, the quantity of LF decreased by $18 \%$. Moreover, the quantity of thermo-LF in mitochondrial suspension was reduced even more with a much smaller number of glutathione $(100 \mu \mathrm{M})$-by $71 \%$. It can be assumed that at high concentration $(1 \mathrm{mM})$ the molecules of glutathione can interact with each other and this oxidation leads to the formation of the S-S bonds between two molecules, whereas at a low concentration $(100 \mu \mathrm{M})$ most of the thiol groups of glutathione react with Schiff bases of LF.

Glutathione precursor cysteine also has a sulfhydryl group. Therefore, we suggested that cysteine could also be able to cleave the LF. Indeed, the amount of thermal-LF in mitochondria decreased by $25 \%$ after 1 hour incubation with $1 \mathrm{mM}$ cysteine at $23^{\circ} \mathrm{C}$.

Dithiothreitol has two thiol groups with redox potential of $-0.33 \mathrm{~V}$ at $\mathrm{pH} 7$ [28]. It is a strong reducing agent, so it is also able to cleave the Schiff bases. The standard reduction potential of the Schiff bases is in the range of $-1.5 \mathrm{~V}$ to $-2.5 \mathrm{~V}$ [29]. The electromotive force of the reaction is directed towards the reduction of the Schiff bases. In our experiments, the LF concentration decreased by $15 \%$ after incubation of mitochondria with $1 \mathrm{mM}$ dithiothreitol for 1 hour at $23^{\circ} \mathrm{C}$.

Thus, the proposed method allows to obtain the thermo-LF in mitochondrial suspension by prolonged moderate heating and to test a number of compounds as activators, inhibitors and cleavers of this LF. It creates a possibility to study the mechanisms of action of antioxidants and reducing agents relating to prevention of the LF formation. Moreover, this method allows to find a suitable antioxidant concentration in the experimental conditions. It is very important to investigate the influence of different active substances on the process of direct (non-enzymatic) cleavage of LF in vitro (Figure 3).

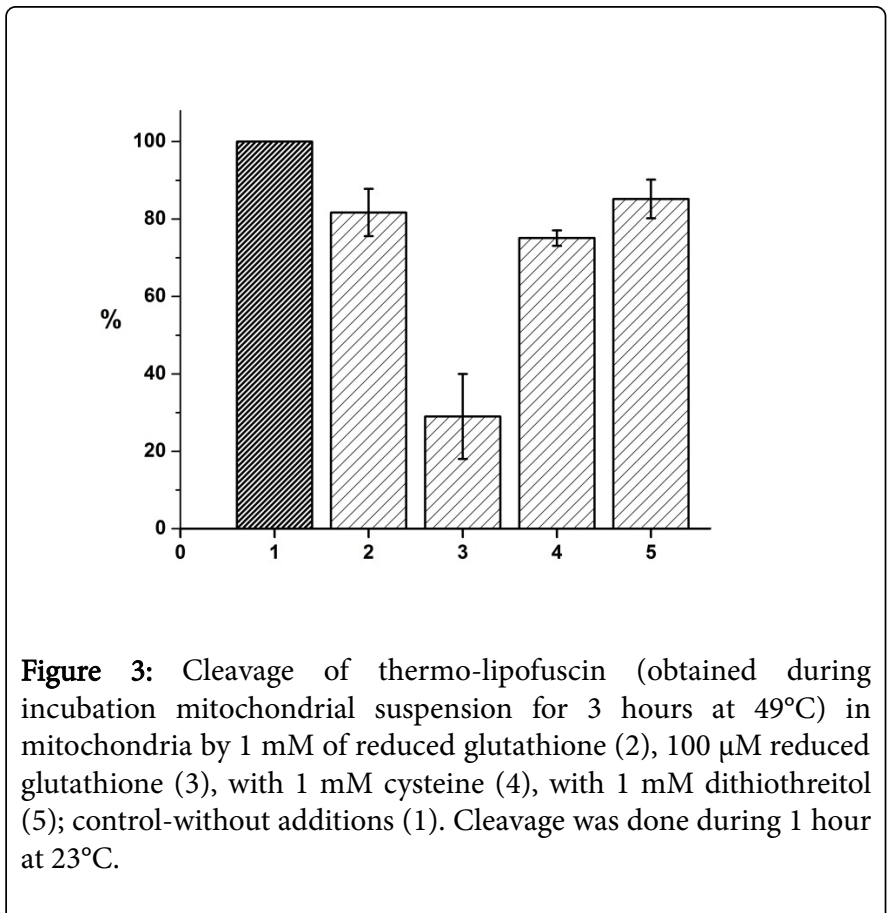

\section{References}

1. Höhn A, Grune T (2013) Lipofuscin: formation, effects and role of macroautophagy. Redox Biol 1: 140-144.

2. Brunk UT, Terman A (2002) The mitochondrial-lysosomal axis theory of aging: accumulation of damaged mitochondria as a result of imperfect autophagocytosis. Eur J Biochem 269: 1996-2002.

3. Jung T, Bader N, Grune T (2007) Lipofuscin: formation, distribution, and metabolic consequences. Annals N Y Acad Sci 1119, 97-111.

4. Terman A, Brunk UT (1998) Lipofuscin: mechanisms of formation and increase with age. APMIS 106: 265-276.

5. Terman A, Brunk UT (1998) On the degradability and exocytosis of ceroid/lipofuscin in cultured rat cardiac myocytes. Mech Ageing Dev 100: 145-156.

6. Donato H, Sohal RS (1978) Age-related changes in lipofuscin-associated fluorescent substances in the adult male housefly Musca domestica. Exp Gerontol 13: 171-179.

7. De Freitas V, da Silva Porto P, Assunçao M, Cadete-Leite A, Andrade JP, et al. (2004) Flavonoids from grape seeds prevent increased alcoholinduced neuronal lipofuscin formation. Alcohol Alcohol 39: 303-311.

8. Shen LR, Parnell LD, Ordovas JM, Lai CQ (2013) Curcumin and aging. Biofactors 39: 133-140.

9. Marzabadi, MR, Sohal RS, Brunk UT (1990) Effect of alpha-tocopherol and some metal chelators on lipofuscin accumulation in cultured neonatal rat cardiac myocytes. Anal Cell Pathol 2: 333-346.

10. Fattoretti P, Bertoni-Freddari C, Casoli T, Di Stefano G, Solazzi M, et al. (2002) Morphometry of age pigment (lipofuscin) and of ceroid pigment deposits associated with vitamin E deficiency. Arch Gerontol Geriatr 34: 263-268.

11. Abdel Mohsen MM, Iravani MM, Spencer JP, Rose S, Fahim AT, et al. (2005) Age-associated changes in protein oxidation and proteasome activities in rat brain: modulation by antioxidants. Biochem Biophys Res Commun 336: 386-391.

12. Kasperczyk S, Dobrakowski M, Kasperczyk J, Ostałowska A, ZalejskaFiolka J, et al. (2014) Beta-carotene reduces oxidative stress, improves glutathione metabolism and modifies antioxidant defense systems in lead-exposed workers. Toxicol Appl Pharmacol 280: 36-41. 
Citation: Vekshin NL, Frolova MS (2018) Formation and Destruction of Thermo-Lipofuscin in Mitochondria. Biochem Anal Biochem 7: 357. doi: 10.4172/2161-1009.1000357

Page 5 of 5

13. Arivazhagan P, Panneerselvam C (2004) Alpha-lipoic acid increases Na $+\mathrm{K}+\mathrm{ATPase}$ activity and reduces lipofuscin accumulation in discrete brain regions of aged rats. Ann N Y Acad Sci 1019: 350-354.

14. Klopstock T, Elstner M, Bender A (2011) Creatine in mouse models of neurodegeneration and aging. Amino Acids 40: 1297-1303

15. Gao G, Ollinger K, Brunk UT (1994) Influence of intracellular glutathione concentration of lipofuscin accumulation in cultured neonatal rat cardiac myocytes. Free Radic Biol Med 16: 187-194.

16. Sharova IV, Vekshin NL (2004) Rotenone-insensitive NADH oxydation in mitochondrial suspension occurs by $\mathrm{NADH}$ dehydrogenase of respiratory chain fragments. Biophys 49: 814-821.

17. Vekshin NL (2009) Photonics of Biopolymers. Springer.

18. Vekshin NL (2009) Fluorescence Spectroscopy of Bio macromolecules. Photon-vek, Pushchino.

19. Schutt F, Ueberle B, Schnölzer M, Holz FG, Kopitz J (2002) Proteome analysis of lipofuscin in human retinal pigment epithelial cells. FEBS Lett. 528: 217-221.

20. Höhn A, Jung T, Grimm S, Grune T (2010) Lipofuscin-bound iron is a major intracellular source of oxidants: role in senescent cells. Free Radic Biol Med 48: 1100-1108.

21. Frolova MS, Surin AM, Braslavski AV, Vekshin NL (2015) Degradation of mitochondria to lipofuscin upon heating and Illumination. Biophys 60: 1125-1131.

22. Babizhayev MA (2010) New concept in nutrition for the maintenance of the aging eye redox regulation and therapeutic treatment of cataract disease; synergism of natural antioxidant imidazole-containing amino acid-based compounds, chaperone, and glutathione boosting agents: a systemic perspective on aging and longevity emerged from studies in humans. Am J Ther 17: 373-389.

23. Wade AM, Tucker HN (1998) Antioxidant characteristics of L-histidine. J Nutr Biochem 9: 308-315.

24. Demir S, Güder A, Yazıcılar TK, Çaglar S, Büyükgüngör O (2015) Syntheses, crystallographic, mass-spectroscopic determination and antioxidant studies of $\mathrm{Co}(\mathrm{II}), \mathrm{Ni}(\mathrm{II})$ and $\mathrm{Cu}(\mathrm{II})$ complexes of a new imidazol based Schiff base. Spectrochim Acta A Mol Biomol Spectrosc 150: 821-828.

25. Sokolova IB, Vekshin NL (2008) Release of flavin from the mitochondrial NADH-dehydrogenase complex. Biophys 53: 73-77.

26. Frolova MS, Vekshin NL (2014) Stabilization of NADH-dehydrogenase in mitochondria by guanosine phosphates and adenosine phosphates. J Fluoresc 24: 1061-1066.

27. Steinkühler C, Pedersen JZ, Weser U, Rotilio G (1991) Oxidative stress induced by a di-Schiff base copper complex is both mediated and modulated by glutathione. Biochem Pharmacol 42: 1821-1827.

28. O'Neil MJ (2001) Merck Index: an encyclopedia of chemicals, drugs, \& biologicals: (13thedn), United States: MERCK \& CO INC.

29. Isse AA, Gennaro A, Vianello E (1997) Electrochemical reduction of Schiff base ligands H2salen and H2salophen. Electrochimica Acta 42: 2065-2071. 